\title{
Nuclear Expression and DNA Binding Capacity of Receptor for Advanced Glycation End Products in Renal Tissue
}

\author{
Brooke E. Harcourt ${ }^{1,2,3}$, Aaron D. McClelland ${ }^{2}$, Hiroshi Yamamoto ${ }^{4}$, Hideto Yonekura ${ }^{4}$, Yasuhiko \\ Yamamoto $^{4}$, Sally A Penfold ${ }^{2}$, Amelia K. Fotheringham ${ }^{1}$, David A. Vesey, 1b,5, David W. \\ Johnson $^{16,5}$, Melinda T. Coughlan², Mark E. Cooper ${ }^{2}$, Phillip Kantharidis ${ }^{2}$, Josephine M. \\ Forbes ${ }^{1,2,6}$
}

\section{Affiliations:}

1. $\quad{ }^{1 \mathrm{a}}$ Glycation and Diabetes, Mater Research and

${ }^{1 b}$ Centre for Kidney Disease Research - University of

Queensland, Translational Research Institute,

Woolloongabba, Queensland, Australia.

2. Baker IDI Heart and Diabetes Institute, Melbourne, Victoria, Australia.

3. Murdoch Childrens Research Institute, Melbourne, Victoria, Australia.

4. Department of Biochemistry and Molecular

Vascular Biology, Kanazawa University Graduate

School of Medical Science, Kanazawa 920-8640,

Japan.

5. Department of Nephrology, Princess Alexandra

Keywords: diabetic nephropathy, advanced glycation, Receptor for advanced glycation end products, RAGE, gene regulation, nuclear factor protein, NF-KB

Address correspondence and reprint/publication requests to: $\mathrm{Dr}$ Brooke E. Harcourt, Murdoch Children's Research Institute, C/O The Royal Children's Hospital, 50 Flemington Road, Parkville, VIC 3052. Email: brooke.harcourt@mcri.edu.au

All work in this Biorvix publication remains the sole copyright of the named authors. Hospital, Brisbane Australia.

6. Mater Clinical School, University of Queensland, Brisbane, Australia.

Abstract: The AGER gene encodes for a number of RAGE isoforms, with the membrane bound signal transduction and "decoy" circulating soluble RAGE being the best characterised. Here we demonstrate a novel nuclear isoform of RAGE in mice and human kidney cortex which by cell and size fractionation we determined to be approximately $37 \mathrm{kda}$. This nuclear RAGE isoform is functional and binds to DNA sequences within the upstream 5' promoter region of its own gene, AGER. This binding was shown to be abrogated by mutating the DNA consensus binding sequences during electromobility shift assay (EMSA) and was independent of NF-DB or AP-1 binding. Cotransfection of expression constructs encoding various RAGE isoforms along with $A G E R$ gene promoter reporter-plasmids identified that the most likely source of the nuclear isoform of RAGE was a cleavage product of the nt-RAGE isoform. In obese mice with impaired kidney function, there was increased binding of nuclear RAGE within the A. Region of ager gene promoter with corresponding increases in membrane bound RAGE in renal cells. These findings were reproduced in vitro using proximal tubule cells. Hence, we postulate that RAGE expression is in part, self-regulated by the binding of a nuclear RAGE isoform to the promoter of the AGER gene (encoding RAGE) in the kidney. We also suggest that this RAGE self-regulation is altered under pathological conditions and this may have implications for chronic kidney disease. 


\section{Introduction}

The receptor for advanced glycation end products (RAGE) is a pattern recognition, multi-isomeric member of the immunoglobulin superfamily. RAGE is widely expressed and contributes to inflammation, diabetes and its complications, cancer, Alzheimer's disease and a number of immunological conditions [1]. The RAGE gene (AGER/ager) is located in the Class III region of the major histocompatibility complex and encodes for endogenous secretory and membrane bound isoforms of RAGE, as well as numerous other tissue specific splice variants yet to be fully characterised [2-4]. In particular, the kidney is thought to express a high number of RAGE splice variants when compared with other organs, of which approximately $50 \%$ are thought to be targeted for degradation, but the function of the remaining splice variants remains unknown $[3,5]$. Full length RAGE (fl-RAGE) represents a membrane spanning isoform which binds complex high molecular weight ligands including advanced glycation end products (AGEs), s100 calgranulins, beta amyloid and DNA bound high mobility group box protein-1 [6]. Ligation with fl-RAGE activates intracellular signalling pathways such as nuclear factor kappa B (NF-DB), [7] and JNK and JAK/Stat pathways, directly impacting on the transcription of many inflammatory genes.

Chronic increases in RAGE signalling in transgenic mice result in renal pathology
[8], particularly in the context of stressors such as diabetes [9]. Blockade of RAGE expression or signalling has also been shown to prevent nephropathy in experimental models of diabetes [10-13], non-diabetic kidney disease [14-16] and obesity [17]. Not surprisingly, administration of SRAGE, a competitive decoy of RAGE is renoprotective in experimental models of chronic kidney disease. Circulating levels of sRAGE but not es-RAGE are predictors of albuminuria in diabetes [18], and are increased with obesity $[19,20]$.

Previous work from Tanji et al, performed using human renal biopsies presented photomicrographs that suggested the presence of nuclear RAGE [15]. Hence, the aim of the present study was to characterise this nuclear isoform of RAGE in the kidney and determine its relevance to renal pathology.

\section{Results}

\section{RAGE deficient mice are protected from high fat induced renal dysfunction}

Here it is demonstrated that RAGE deficiency afforded protection against the development of high fat induced renal injury. The rate of urinary albumin excretion was significantly increased with high fat feeding in wild type mice, but not in RAGE deficient mice (Figure 1A: AER). Similarly, creatinine clearance was increased in wildtype mice that were fed high fat diets indicative of renal hyperfiltration, and this was ameliorated in mice with RAGE deficiency (Figure 1B: $\mathrm{CrCl}$ ).

All work in this Biorvix publication remains the sole copyright of the named authors. 
A

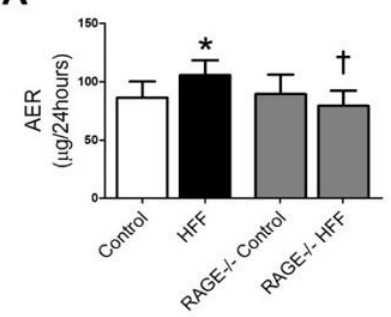

B

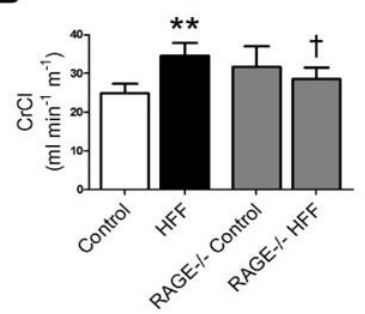

Figure 1: Renal Function in WT and RAGE Deficient (RAGE-/-) Mice

A: Urinary Albumin Excretion Rate (AER

$\mu \mathrm{g} / 24$ hours) by ELISA B: Creatinine clearance

$\left(\mathrm{CrCl} \mathrm{ml} / \mathrm{min} / \mathrm{m}^{2}\right)$ by HPLC.

High Fat Fed (HFF). ${ }^{*} p<0.05$ vs Control,

${ }^{* *} p<0.01$ vs Control, $+p<0.05$ vs HFF

\section{Nuclear RAGE expression is present in renal cortices}

Membrane bound RAGE expression (fIRAGE) was increased within renal cortices taken from obese mice with renal impairment (Figure 2A). For the first time, the presence of RAGE was also demonstrated within cell nuclei, from renal cortices of all wild type (WT) mice (Pictured Figure 2B-D), and this was further increased in obese high fat fed WT mice.

The presence of nuclear RAGE was confirmed in human nephrectomy tissues obtained from a healthy donor (Figure 2E), as well as within the renal cortex from an overweight individual with type 2 diabetes and nephropathy (Figure 2F).

Endogenous secretory RAGE (es-RAGE), which is also transcribed from ager/AGER, and is part of the circulating soluble RAGE pool (sRAGE), where the remaining sRAGE is contributed from membrane cleavage from endothelial cell surfaces [21]. With obesity, circulating soluble RAGE concentrations were increased as compared with control mice (Figure 2G).

Immunoblotting for RAGE in ultrafractionated nuclear extracts prepared from renal cortices of control and obese mice, identified a single $37 \mathrm{kDa}$ sized protein isoform, that was not identified in the other cellular fractions (Figure $2 \mathrm{H}$ : Lane 1-4). Immunoblotting for RAGE in the membrane fractions of kidney cortices from these mice showed full length $(50 \mathrm{kDa})$ and endogenous secretory RAGE immunoreactivity (25kda, Figure $2 \mathrm{H}$ ), consistent with previous studies.

\section{Expression of Nuclear RAGE is inducible}

Given the observation of RAGE protein within the nucleus, the capacity for RAGE to bind DNA was next examined using reporter constructs that contained known sequences of the AGER 5'promoter region to determine whether self-regulation of RAGE was occurring [22]. Indeed, RAGE has been reported to bind double stranded DNA in vitro (dsDNA, [23]) and CpG oligonucleotides in systemic lupus nephritis [24], although the reason for this is unknown. In the present study, rat proximal tubular cells were transiently transfected with a variety of human expression constructs to overexpress the known RAGE protein isoforms, es-RAGE, fIRAGE, as well as N-truncated RAGE (ntRAGE). These were co-transfected with three different luciferase reporter constructs containing various known DNA binding elements within the AGER 5' promoter region ([22]; Model Figure 3A).

All work in this Biorvix publication remains the sole copyright of the named authors. 

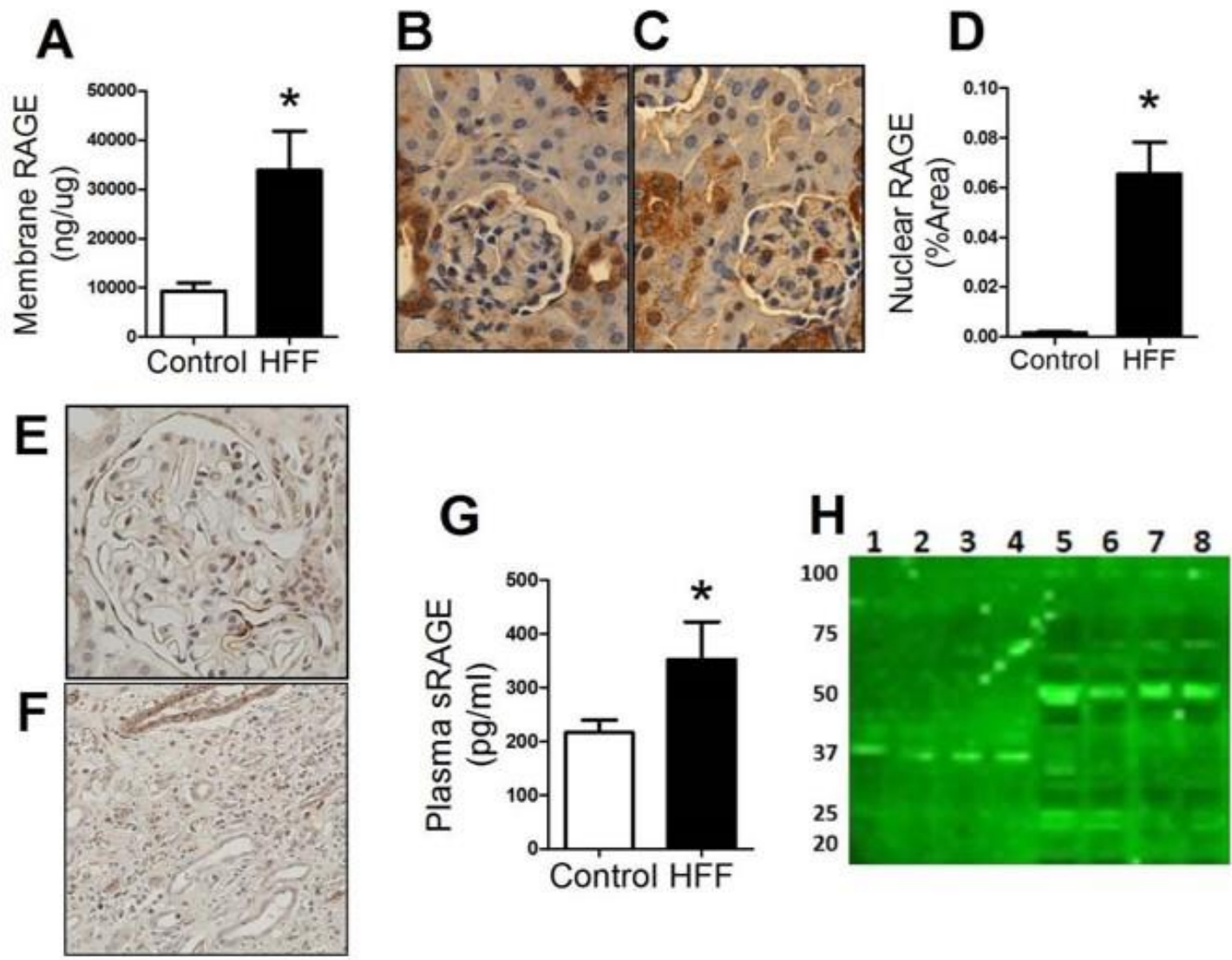

Figure 2: Expression of RAGE Isoforms

A: RAGE expression in membrane isolates from renal cortices of wild type Control and wild type HFF groups, as measured by ELISA. Representative immunohistochemical micrographs for B. WT Control mice, and C: WT HFF mice D: Quantification of RAGE positive stained nuclei as a percentage of total area, from WT Control and WT HFF mice. E: Representative micrographs for RAGE immunoactivity in Non-diabetic human renal nephrectomy. F: A human renal biopsy sample taken from an obese individual with T2DM. G: Quantification of soluble RAGE isoforms (sRAGE and esRAGE) in plasma from WT Control and HFF mice. H: Western immunoblot analysis of renal cortical fractions from WT mice, NUCLEAR (LANE 1-4) and MEMBRANE (LANE 5-8). High Fat Fed (HFF).

$* p<0.05$ Control vs HFF

Firstly, transient overexpression of either flRAGE or nt-RAGE, co-transfected with the pGL-1 reporter, demonstrated an increase in AGER transcription, which was also seen following exposure to an inducer of RAGE transcription, TNF- $\alpha$ [22]. This region contains binding sites for Sp-1, AP-1 and NF$\square B$ and activation of these transcription factors has been shown following RAGE ligation [25-27]. There was also robust induction of $A G E R$ transcription reported by the pGL-5 construct which contains NF-DB binding elements (nucleotides -751-0), following cotransfection with any RAGE overexpressing plasmid (Figure 3B-D).

All work in this Biorvix publication remains the sole copyright of the named authors. 
bioRxiv preprint doi: https://doi.org/101101/632596; this version posted May 9, 2019. The copyright holder for this preprint (which was not certified by peer review) is the author/funder, who has granted bioRxiv a license to display the preprint in perpetuity. It is made available under aCC-BY-NC-ND 4.0 International license.

Harcourt et al 2019

Renal Nuclear RAGE

A

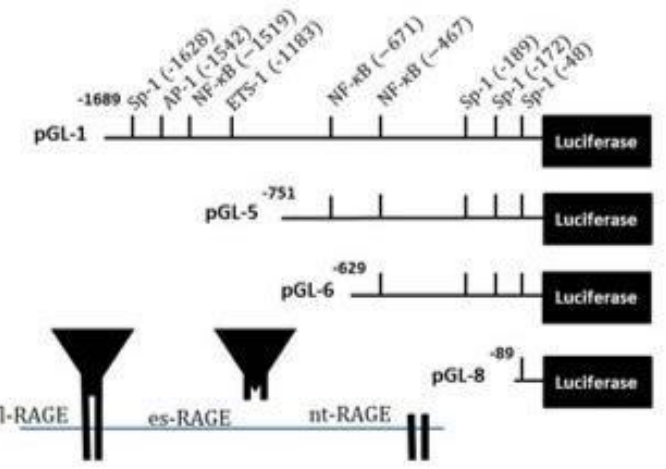

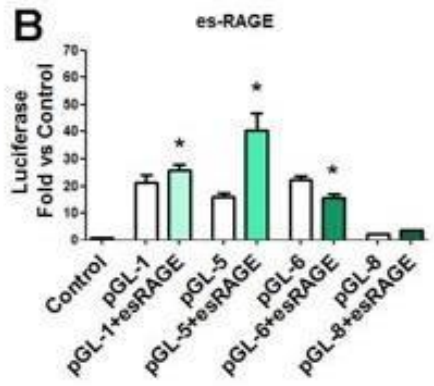

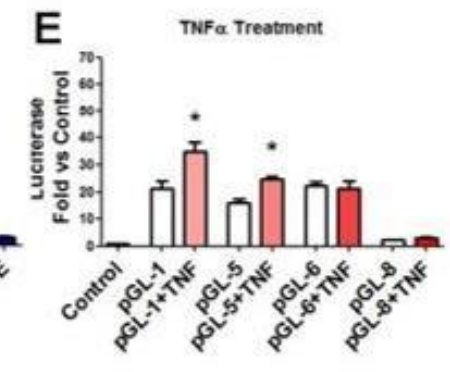

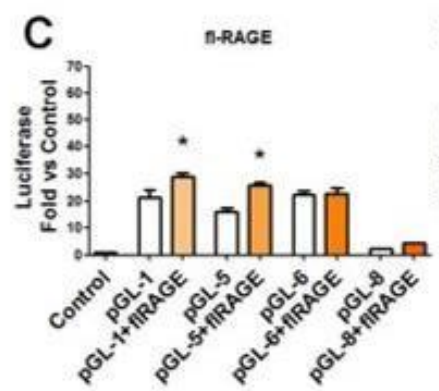
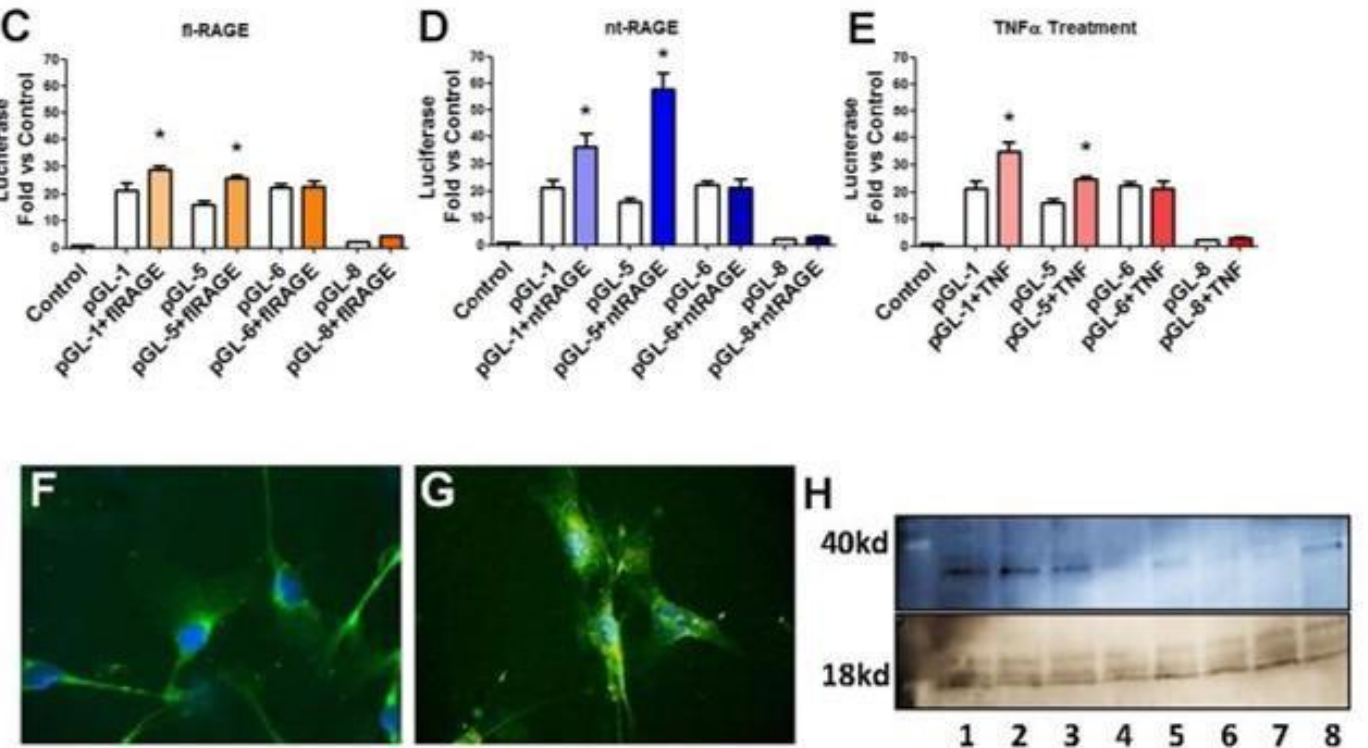

Figure 3: Inducible Expression of Nuclear RAGE

A: Model of luciferase reporter plasmids transfected into renal cell line containing various regions of the AGER gene (which encodes RAGE), to test transcription. b-d These reporter plasmids (PGL-1, PGL-5, PGL- 6 or PGL-8) were cotransfected with plasmids containing CDNA to overexpress various RAGE isoforms. : B: Luciferase reporter plasmids for the $5^{\prime}$ promoter region of AGER co-transfected with es-RAGE over-expressing plasmid. Results represented as fold-change from Control. C: Luciferase reporter plasmids for the $5^{\prime}$ promoter region of AGER co-transfected with fl-RAGE over-expressing plasmid. Results represented as foldchange from Control. D: Luciferase reporter plasmids for the 5' promoter region of AGER co-transfected with nt-RAGE over-expressing plasmid. Results represented as fold-change from Control. E: Luciferase reporter plasmids for the $5^{\prime}$ promoter region of AGER and treated with the AGER gene transcriptional activator TNF- $\alpha$. Results represented as fold-change from Control. f-g Confocal micrographs of RAGE immunofluorescence (green) (using an antibody against the C-terminus of RAGE) nuclear staining (blue), in primary renal mesangial cells treated with F. Unmodified albumin or G. AGE modified albumin (AGE-BSA where nuclear staining is evident (green)), H: Western immunoblot of nuclear fraction of RAGE ( $\sim 37 \mathrm{kDa}$ ) from cells transfected with RAGE isoform over-expressing plasmids and histone-3 (16kda): LANE1-2 nt-RAGE, LANE 3-4 es-RAGE, LANE 5$6 \mathrm{fl}$-RAGE, LANE 7-8 Control plasmid.

${ }^{*} p<0.05$ single transfection of plasmid vs double transfection of respective plasmid (white bar vs colour bar)

This suggested that all of these RAGE isoforms have the capacity to bind within this $A G E R$ promoter region and induce RAGE transcription. It was previously shown that exposure of endothelial cells to AGE-BSA also led to DNA binding within the AGER promoter in regions upstream of that contained in the pGL-6 construct [22]. This suggested that RAGE ligands, such as AGE modified albumin, may potentially stimulate nt-RAGE or fl-RAGE production and translocation to the nucleus, as was

All work in this Biorvix publication remains the sole copyright of the named authors. 
bioRxiv preprint doi: https://doi.org/101101/632596; this version posted May 9, 2019. The copyright holder for this preprint (which was not certified by peer review) is the author/funder, who has granted bioRxiv a license to display the preprint in perpetuity. It is made available under aCC-BY-NC-ND 4.0 International license.
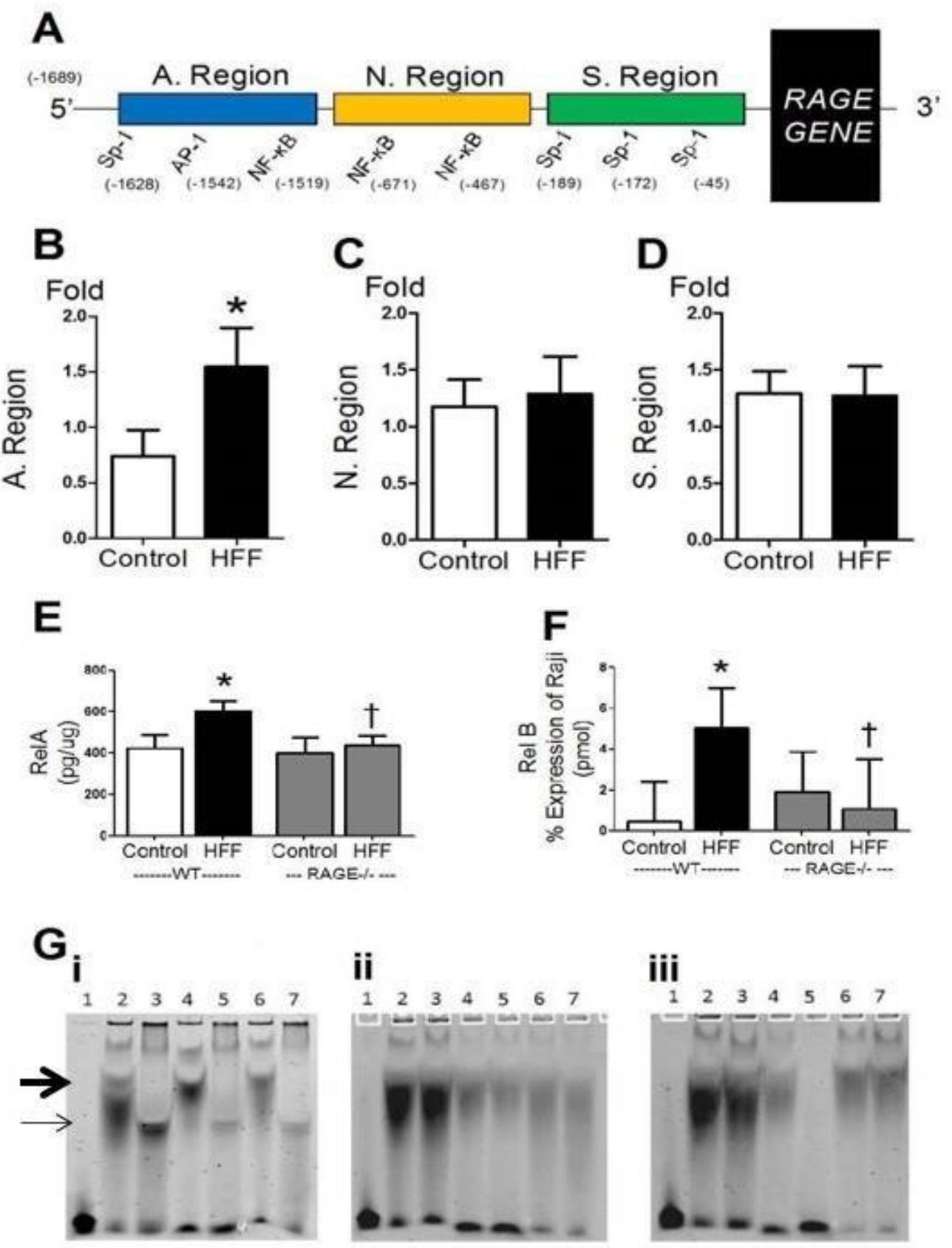

Figure 4: AGER (RAGE gene) Promoter Region Interaction and Transcription

A: Model of chromatin immunoprecipitation (ChIP) quantitative RT-PCR probe and primer design regions. A. Region (-1628 to-1519), N. Region (-671 to-467) and S. Region ( -189 to -45$)$ b-d Chromatin immunoprecipitation of RAGE on DNA from kidney cortices of WT Control and HFF mice. B: Interaction of RAGE with chromatin in the A. Region of the $5^{\prime}$ promoter region of ager. C: Interaction of RAGE with chromatin in the N. Region of the 5' promoter region of ager. D: Interaction of RAGE with chromatin in the S. Region of the $5^{\prime}$ promoter region of RAGE. E: Total RelA DNA binding activity in renal cortices from WT and RAGE deficient mice. F: Total RelB DNA activity in renal cortex from WT and RAGE deficient mice. G: Electromobility shift assay (EMSA) of specific oliogonucleotide regions of the A. Region of the ager promoter in kidney cortex DNA from control mice demonstrating i) RAGE antibody Supershift, ii) Sp-1 antibody Supershift, iii) NF- $k B$ antibody Supershift. Lane 1. No DNA Control. Lane 2. Construct 1. Lane 3. Construct 1. with Antibody Supershift, Lane 4. Construct 2. Lane 5. Construct 2 with Antibody Supershift, Lane 6 . Construct 3, Lane 7. Construct 3 with Antibody Supershift. H: Interaction of ER- $\beta$ bound chromatin in N. Region of the $5^{\prime}$ promoter region of the RAGE gene. I: Interaction of ER- $\beta$ bound chromatin in S. Region of the $5^{\prime}$ promoter region of the RAGE gene.

High Fat Fed (HFF). RAGE deficient (RAGE- $/-$ ) ${ }^{*} p<0.05$ Control vs HFF, $+p<0.05$ WT HFF vs RAGE- $/$ - HFF

All work in this Biorvix publication remains the sole copyright of the named authors. 
seen in mesangial cells in the present study in response to AGE-albumin (Figure $3 \mathrm{~F}$ and G). There was however, no change in reporter activity seen with either the pGL-6 (-629-0) or pGL-8 (-89-0) reporter constructs of the AGER promoter when cotransfected with plasmids overexpressing fl-RAGE or ntRAGE. These data suggest that the $\mathrm{fl}$ and ntRAGE derived isoforms may not bind within this region of the AGER promoter (-629-0). However, transient transfection to overexpress esRAGE when co-transfected with the pGL-6 reporter construct of the 5' AGER promoter (-629-0; Figure 3B), significantly decreased transcription, in contrast to that seen with both fl-RAGE (Figure $3 \mathrm{C}$ ) and ntRAGE

(Figure 3D).

Furthermore, using western immunoblotting, it appeared that overexpression of nt-RAGE (Lanes 1-3; Figure $3 \mathrm{H}$ ) resulted in elevated nuclear RAGE (Lanes 1-2) when compared with either fl-RAGE (Lanes 5-6. Figure 3H) or esRAGE (Lanes 3-4; Figure 3H).

\section{Chromatin interaction of nuclear RAGE in the 5' promoter region of AGER}

Given that other transcription factors have previously been identified following the binding of ligands to membranous fl-RAGE [25-27], the association of these factors with the binding of the nuclear RAGE isoform to DNA was examined. Using chromatin immunoprecipitation (ChIP) we identified that RAGE was bound to chromatin taken from nuclei of renal cortices of both control and obese mice (Figure 4A). RAGE bound to chromatin was increased in the A. Region (nucleotides 1628 to -1519 ) of the ager 5' promoter region within renal tissue from obese mice (Figure 4A). This region also contained Sp1, AP-1 and NF-DB transcriptional binding elements. Whilst, RAGE bound chromatin within both the N. and S. Regions (Figure 4A-D) of the ager promoter, this was not different among WT and obese mouse groups. Nuclear translocation and DNA binding of Rel $A$ (Figure 4E) and Rel B (Figure 4F) complexes of NF-CB, were also increased in kidney cortices with obesity, and were reduced in the kidneys of RAGE deficient obese mice (RAGE-/-).

Using oligonucleotide sequences spanning the A. Region of the ager promoter in electromobility shift assays (EMSA) where increased chromatin binding of RAGE was detected during the ChIP analysis, protein interaction was further analysed. Increased DNA binding activity when the nucleotide sequence CACCCC with supershifting was confirmed using RAGE antibody (Figure 4Gi). This was confirmed using mutant oligonucleotide sequences (Suppl Figures 1A). Absence of DNA binding or antibody supershift was observed for Sp-1 and NFCB (Figure 4Gii and iii).

Previous studies [22, 28], have also described the interaction of nuclear estrogen receptor ER- $\alpha$ with the AGER promoter region in human endothelial cells. Hence, the binding of ER- $\alpha$ and ER- $\beta$ to chromatin was next investigated using ChIP, in renal cortices of WT and RAGE-/- mice

All work in this Biorvix publication remains the sole copyright of the named authors. 
with and without obesity. There were no differences seen between groups when ChIP for ER- $\alpha$ was examined within the ager promoter (Suppl Figures 1B-C), despite DNA binding of ER- $\alpha$ in all mouse groups. However, using ChIP, nuclei from renal cortices showed that estrogen receptor- $\beta$ (ER- $\beta$ ) DNA binding activity within both the $\mathrm{N}$. and $\mathrm{S}$. regions of the ager promoter increased with obesity as compared with WT mice. This was not observed in RAGE-/obese mice (Figure

$4 \mathrm{H}-\mathrm{I})$.

\section{Discussion}

For the first time, this study has demonstrated a novel nuclear isoform of RAGE in renal cortex and identified binding sites for nuclear translocated within the ager gene. The expression of nuclear RAGE was inducible by known ligands in vitro, and differentially by RAGE isoforms and was altered in a murine model of high fat feeding. The nuclear RAGE isoform was additionally identified in human nephrectomies.

The presence of RAGE in the nuclei of renal cortical cells is novel finding. Previously, this particular isoform may have been overlooked to the use of crude whole cell or tissue homogenisation and protein lysate analysis. However, some previous studies have suggested the presence of an additional functional RAGE isoform, with unknown localisation and function. Hudson et al, reported a $\sim 40 \mathrm{kDa}$ RAGE protein isoform [2], which was produced as a result of genetic recombination of ager, but the cellular location of that isoform was not determined in that study. A RAGE isoform of $\sim 40 \mathrm{kDa}$ was also described in total cell lysates of neuroblastoma cells [29], as well as peripheral blood mononuclear cells [30] but again the function and subcellular localisation of this isoform has remained undetermined. A similar sized functional protein was also identified by Kalea et al [3], in mice as a result of genetic recombination of $A G E R$. In these studies the newly identified isoform of RAGE was not fully characterised for cellular location of function. These findings along with our present observations in the kidney suggest that further research into the function of this RAGE isoform and its effects on the transcription of other genes is warranted.

From the present study, it appears that already characterised isoforms of RAGE have varying effects on the transcription of the RAGE gene (ager). For example, overexpression of nt-RAGE, which is present of cell membranes but does not bind ARGE ligands, resulted in both increased RAGE transcription in reporter assay and increased expression of the nuclear RAGE isoform. Using specific oligonucleotide sequences in EMSA, the binding sites of nuclear RAGE in the AGER promoter were able to be characterised in renal tissue. In addition, supershift assay suggested that this nuclear RAGE was not acting by binding to known transcription factors to regulate AGER within this DNA region as distinct from other regions of the ager promoter. Indeed,

All work in this Biorvix publication remains the sole copyright of the named authors. 
there was no difference between control and high fat fed mice on ChIP for RAGE biding in regions other than the $A$ region of the ager promoter. Other identified sites affecting ager transcription, were consistent with those previously identified for the AGER [31] Hence it is tempting to speculate that the nt-RAGE isoform, which in renal cells can be seen on the cell membrane surface but does not bind ligands and has an as yet uncharacterised role [4] is the major source of nuclear RAGE. Further, it is also suggested that this nuclear RAGE uniquely interacts with a binding site located within the $A$. Region of the ager promoter affecting the expression of RAGE in pathologies such as seen with obesity in this model. It was further identified that NF-CB and SP-1 remained unchanged in this region of the promoter.

Of interest, was the observation that cellular over-expression of other RAGE isoforms, such as membrane bound RAGE and esRAGE, also caused transcription or suppression of ager gene transcription respectively. However, the over-expression of these particular isoforms did not appear to increase the appearance of RAGE in the nucleus which suggests that these RAGE isoforms likely caused effects on AGER via other transcription factors. Indeed, a number of previous studies, including our own $[32,33]$ have shown changes in gene transcriptional regulation via NF-KB and other transcription factors as was also seen in the present study for the NF-KB subunits, RelA and RelB. Interestingly, however AGER transcription induced by es-RAGE (nucleotides -629 to 0) was supressed in the present study. This is consistent with the thinking that soluble RAGE may regulate the expression or signal transduction of full length membranebound $\operatorname{RAGE}[34,35]$, although the exact mechanism by which this occurred was beyond the scope of the present study. Taken together the findings highlight a complex mechanism of AGER gene regulation that has not been previously described.

The finding that RAGE can be found within nuclei and that it has direct binding activity within its own promoter lays foundations for the possibility that nuclear RAGE, most likely in association with proteins, such as NF-CB may play a regulatory role in the transcription of $A G E R$ and potentially other genes. There is also evidence provided within the present study that dysregulation of RAGE binding within its own promoter may occur during renal disease, contributing to pathology development and progression. These findings could directly translate to other diseases where RAGE is implicated as a pathogenic mediator.

\section{Methods}

\section{Mouse Model}

Male wild-type, C57BL/6J and RAGEdeficient (RAGE-/-, [14] backcrossed 9 times onto C57BL/6J) mice, were randomised to either control (AIN-93G, Specialty Feeds, Perth, Australia), or high fat diets (SF05-031, Specialty Feeds, Perth, Australia) diet for 16 weeks which resulted in significant weight

All work in this Biorvix publication remains the sole copyright of the named authors. 
gain (as previously described Control $2.9 \pm 2.4 \mathrm{~g}$ vs high fat fed

$11.0 \pm 1.6 \mathrm{~g}, p<0.05$, RAGE-/- Control $8.3 \pm 2.1 \mathrm{~g}$ vs $15.4 \pm 2.2 \mathrm{~g}, \quad p<0.01[17])$. Mice were monitored weekly for body weight and plasma glucose concentrations. Mice were housed in a temperate environment with a 12 hour light-dark cycle with ad libitum access to food and water. All studies were performed in accordance with the guidelines from the National Health and Medical Research Council of Australia and the AMREP Ethics Committee.

Renal function was determined using 24 hour urine and plasma collections at the study endpoint. Creatinine clearance $(\mathrm{CrCl})$ and urinary albumin excretion rate (UAER) were determined, as previously described [11].

\section{Human Kidney Sections}

Paraffin embedded renal sections were obtained from Clay Winterford (QIMR, Queensland, Australia). Normal renal tissue was obtained from patients undergoing partial nephrectomy for solid tumours.

\section{RAGE Protein Analysis}

Kidneys were removed and immediately frozen in liquid nitrogen. The medulla was removed and the cortex was subjected to an ultrafractionation protocol that separated the cortex into membrane, cytosolic and the nuclear fractions, as previously described [11].

Cytosolic, membrane and nuclear protein fractions were analysed via ELISA (RAGE, Quantikine, R\&D, Minneapolis, MN, USA).
The protein concentrations of the ELISA were corrected for the total protein concentrations of the sample as determined by Qubit fluorescence incorporation protocol (Qubit, Life Technologies, CA, USA).

Twenty-five micrograms of each renal cortex fraction was added to $10 \%$ or $12 \%$ polyacrylamide gels (Mini-protean TGX precast gels, Bio-rad, CA, USA), and assessed using infrared secondary probes (LICOR, NA, USA).

Immunohistochemistry analysis for RAGE protein was performed in paraffin embedded human and mouse kidney sections, as previously described [11].

Western immunoblotting was performed as previously described [11]. Briefly, 25ug of nuclear or membrane/cytosolic fractions from WT and WT high fat fed mice, were subjected to SDS-PAGE and western immunoblotting with rabbit anti-Cterminal RAGE (a kind gift from Prof M. Neeper, Novo Nordisk Industries).

Primary mouse mesangial cells grown on glass coverslips were exposed to AGE-BSA or BSA (100ug/mL [36], 72 hours) in normal growth media (DMEM, Invitrogen, CA, USA) Following fixation with cold methanol and blocking with $1 \%$ BSA, presence of RAGE in the nucleus was confirmed using immunofluorescence (primary antibody, 1:200 goat anti-RAGE (Millipore, CA, USA), 1:5000 secondary antibody donkey antigoat 488 (LICOR, NA, USA)). Cells were mounted using fluroshield with DAPI mounting media (Sigma-Alderich, CA, USA)

0 All work in this Biorvix publication remains the sole copyright of the named authors. 
and imaged on an Olympus Xcellence Pro confocal microscope (20x magnification).

\section{Chromatin Immunoprecipitation (ChIP)}

Kidney cortices were analysed for activity in the $5^{\prime}$ flanking region of the ager (RAGE) gene. Probes were designed using BLAST and were created to allow specific analysis of whether RAGE, ER- $\alpha$ or ER- $\beta$ activity was able to directly influence the up-regulation of the RAGE gene. The primers designed also allowed for specific identification of the region of RAGE promoter that was bound. Promoter A. Region (-1628 to1519), Probe; TCTGGAGATGTCAGCCC with an MGB quencher (Taqman, Invitrogen,

USA), Forward primer;

GTTCCCCACCCCACTTATATACTCT, Reverse primer; TCCCCATTTTTTGGCATCTCT.

Promoter N. Region (-671 to-467), Probe;

CCCTCAGACACATCCTC with a 3' MGB quencher (Taqman, Invitrogen), Forward primer; CAGCCCTGAACCCTTCATCTG,

Reverse primer;

CCCATGgtgacAgtCTtGaAgA. Promoter S. Region (-189 to -45 . Probe; ACCTGAAGGACTCTTG with an MGB quencher (Taqman, Invitrogen), Forward primer; GGTCGGGTGAGATTGCTTCTAG, Reverse primer;

TGCCAGGAATCTGTGCTTCTG.

Protein and DNA interactions in renal cortices were fixed in $2 \%$ paraformaldehyde, prior to chromatin extraction and purification. DNA fragments were sheared into $300 \mathrm{bp}$ fragments using Bioruptor sonication at $4{ }^{\circ} \mathrm{C}$ (Ion Torrent, Life
Technologies, NY, USA). Following preclearing with salmon sperm/Protein $A$ agarose slurry (GE Healthcare, NY, USA), 1:12 for 1 hour at $25^{\circ} \mathrm{C}$, chromatin was immunoprecipitated with ER- $\alpha$ (rabbit antiER- $\alpha$, Millipore, CA, USA; 1:100), ER- $\beta$ (rabbit anti-ER- $\beta$, Millipore, CA, USA;

4ug/ul), or RAGE (goat anti-RAGE, Millipore, CA, 1:50) at $4^{\circ} \mathrm{C} 18$ hours with constant rotation. Protein bound DNA antibody interactions were removed with the additional salmon sperm/Protein $A$ agarose. The bound, unbound, and input control DNA samples were purified and analysed. All results were expressed relative to values from WT Control, which were assigned an arbitrary value of 1 . Statistical analysis was performed, and reported on Logtransformed data $(\mathrm{Y}=\log (\mathrm{Y}))$ as data was found to be nonparametric.

\section{Electromobility Shift Assay (EMSA) EMSA}

was performed according to kit instructions (LICOR, NA, USA). Briefly, 10ug nuclear protein was incubated with dsDNA infrared tagged constructs (Table 1. 5'-3' First strand, IDT, NSW, AUSTRALIA) with or without the additional supershift antiRAGE antibody (1ug, Millipore, CA, USA).

Table 1: 5'-3' EMSA dsDNA constructs

\begin{tabular}{|l|l|}
\hline $\begin{array}{l}\text { Construct } \\
\text { 1: Region } \\
\text { A.1. }\end{array}$ & GTTCCCCACCCCACTTATATACTCT \\
\hline $\begin{array}{l}\text { Construct } \\
\text { 2: } \text { Region }\end{array}$ & GAGGCCCTGGGCCAACACCCCCTCC \\
A.2. & \\
\hline $\begin{array}{l}\text { Construct } \\
\text { 3: } \text { Region } \\
\text { A.3. }\end{array}$ & CCATGCACCCCCACCTCCTGC \\
\hline
\end{tabular}

1 All work in this Biorvix publication remains the sole copyright of the named authors. 


\begin{tabular}{|l|l|}
\hline $\begin{array}{l}\text { Construct } \\
\text { 4: Region }\end{array}$ & GTTCCGACGCCACTTATATACTCT \\
A.1M1. & \\
\hline Construct & \\
5: Region & GTTCCCCACGGCACTTATATACTCT \\
A.1M2. & \\
\hline
\end{tabular}

\section{Luciferase Reporter Assay}

Luciferase reporter assays for 5 'regions of the AGER pGL-1, pGL-5, pGL-8, with cotransfection of CDNA plasmids to overexpress fl-RAGE, es-RAGE and nt-RAGE [22] were performed in the mesangial cell line; NRK-52E, in normal glucose conditions for 72 hours. Luciferase assays were performed as previously described [22].

\section{Statistical Analysis}

Results are expressed as Mean $( \pm S D)$ unless otherwise specified. Analyses were performed by ANOVA followed by, Tukey's post-hoc analysis. Unpaired Student's t-test analysis was used to compare means between 2 groups where indicated

(Version 5, Graphpad Software, L Jolla, CA, USA). A $p<0.05$ was considered to be statistically significant.

\section{Disclosure}

The funding bodies (which were NHMRC and JDRF) had no role in the study design, data collection and analysis, decision to publish, or preparation or the manuscript.

\section{Acknowledgements and Declaration}

The authors would like to acknowledge the technical assistance of Domenica Mc Carthy and Maryann Arnstein. We also thank Merlin C. Thomas and Mark A. Febbraio (Baker IDI Heart and Diabetes Research Institute, Melbourne, Victoria, Australia) for their assistance with the project. Further acknowledgement and thanks is paid to Professor Angelika Bierhaus (dec). Sections of paraffin embedded human kidney tissue were supplied by Clay Winterford (Queensland Institute of Medical Research. Brisbane, Queensland, Australia).

Author Contributions: BEH, PK and JMF designed experiments, analysed data, wrote and revised the manuscript. ADM, HYa, HYo, $\mathrm{YH}$ were involved in the design and making of the constructs for the Luciferase Reporter Assays. SAP, AKF and DAM performed experiments. MEC and MTC edited the manuscript.

BEH is a NHMRC Peter Doherty Early Career Fellow, and JMF is an NHMRC

Senior Research Fellow.

2 All work in this Biorvix publication remains the sole copyright of the named authors. 


\section{$\underline{\text { References }}$}

1. Steenvoorden, M.M., et al., RAGE activation induces invasiveness of RA fibroblast-like synoviocytes in vitro. Clinical and experimental rheumatology, 2007. 25(5): p. 740-2.

2. Hudson, B.I., et al., Identification, classification, and expression of RAGE gene splice variants. FASEB journal : official publication of the Federation of American Societies for Experimental Biology, 2008. 22(5): p. 1572-80.

3. Kalea, A.Z., et al., Alternative splicing of the murine receptor for advanced glycation endproducts (RAGE) gene. FASEB journal : official publication of the Federation of American Societies for Experimental Biology, 2009. 23(6): p. 1766-74.

4. Yonekura, H., et al., Novel splice variants of the receptor for advanced glycation end-products expressed in human vascular endothelial cells and pericytes, and their putative roles in diabetes-induced vascular injury. Biochem J, 2003. 370(Pt 3): p. 1097-109.

5. Hudson, B.I., et al., Effects of novel polymorphisms in the RAGE gene on transcriptional regulation and their association with diabetic retinopathy. Diabetes, 2001. 50(6): p. 1505-11.

6. Penfold, S.A., et al., Circulating high-molecular-weight RAGE ligands activate pathways implicated in the development of diabetic nephropathy. Kidney international, 2010. 78(3): p. 287-95.

7. Zong, H., et al., Homodimerization is essential for the receptor for advanced glycation end products (RAGE)-mediated signal transduction. The Journal of biological chemistry, 2010. 285(30): p. 23137-46.

8. D'Agati, V. and A.M. Schmidt, RAGE and the pathogenesis of chronic kidney disease. Nature reviews. Nephrology, 2010. 6(6): p. 352-60.

9. Yamamoto, Y., et al., Receptor for advanced glycation end products is a promising target of diabetic nephropathy. Ann N Y Acad Sci, 2005. 1043: p. 562-6.

10. Yamamoto, Y., et al., Development and prevention of advanced diabetic nephropathy in RAGE- overexpressing mice. J Clin Invest, 2001. 108(2): p. 261-8.

11. Tan, A.L., et al., Disparate effects on renal and oxidative parameters following RAGE deletion, AGE accumulation inhibition, or dietary AGE control in experimental diabetic nephropathy. Am J Physiol Renal Physiol, 2010. 298(3): p. F763-70.

12. Flyvbjerg, A., et al., Long-term renal effects of a neutralizing RAGE antibody in obese type 2 diabetic mice. Diabetes, 2004. 53(1): p. 166-72.

13. Wendt, T.M., et al., RAGE drives the development of glomerulosclerosis and implicates podocyte activation in the pathogenesis of diabetic nephropathy. The American journal of pathology, 2003. 162(4): p. 1123-37.

14. Liliensiek, B., et al., Receptor for advanced glycation end products (RAGE) regulates sepsis but not the adaptive immune response. J Clin Invest, 2004. 113(11): p. 1641-50.

15. Tanji, N., et al., Expression of advanced glycation end products and their cellular receptor RAGE in diabetic nephropathy and nondiabetic renal disease. Journal of the American Society of Nephrology : JASN, 2000. 11(9): p. 1656-66.

16. Ritthaler, U., et al., Expression of receptors for advanced glycation end products in peripheral occlusive vascular disease. The American journal of pathology, 1995. 146(3): p. 688-94.

17. Harcourt, B.E., et al., Targeted reduction of advanced glycation improves renal function in obesity. Kidney international, 2011. 80(2): p. 190-8.

3 All work in this Biorvix publication remains the sole copyright of the named authors. 
18. Humpert, P.M., et al., Soluble RAGE but not endogenous secretory RAGE is associated with albuminuria in patients with type 2 diabetes. Cardiovascular diabetology, 2007. 6: p. 9.

19. Falcone, C., et al., Plasma levels of soluble receptor for advanced glycation end products and coronary artery disease in nondiabetic men. Arteriosclerosis, thrombosis, and vascular biology, 2005. 25(5): p. 1032-7.

20. Koyama, $\mathrm{H} .$, et al., Plasma level of endogenous secretory RAGE is associated with components of the metabolic syndrome and atherosclerosis. Arteriosclerosis, thrombosis, and vascular biology, 2005. 25(12): p. 2587-93.

21. Raucci, A., et al., A soluble form of the receptor for advanced glycation endproducts (RAGE) is produced by proteolytic cleavage of the membrane-bound form by the sheddase a disintegrin and metalloprotease 10 (ADAM10). FASEB journal : official publication of the Federation of American Societies for Experimental Biology, 2008. 22(10): p. 3716-27.

22. Tanaka, N., et al., The receptor for advanced glycation end products is induced by the glycation products themselves and tumor necrosis factor-alpha through nuclear factor-kappa $B$, and by 17 beta-estradiol through Sp-1 in human vascular endothelial cells. The Journal of biological chemistry, 2000. 275(33): p. 25781-90.

23. Park, H., F.G. Adsit, and J.C. Boyington, The 1.5 A crystal structure of human receptor for advanced glycation endproducts (RAGE) ectodomains reveals unique features determining ligand binding. J Biol Chem, 2010. 285(52): p. 40762-70.

24. Ruan, B.H., et al., Complement C3a, CpG oligos, and DNA/C3a complex stimulate IFN-alpha production in a receptor for advanced glycation end product-dependent manner. J Immunol, 2010. 185(7): p. 4213-22.

25. Li, J. and A.M. Schmidt, Characterization and functional analysis of the promoter of RAGE, the receptor for advanced glycation end products. The Journal of biological chemistry, 1997. 272(26): p. 16498-506.

26. Li, J., X. Qu, and A.M. Schmidt, Sp1-binding elements in the promoter of RAGE are essential for amphoterin-mediated gene expression in cultured neuroblastoma cells. The Journal of biological chemistry, 1998. 273(47): p. 30870-8.

27. Bianchi, R., I. Giambanco, and R. Donato, S100B/RAGE-dependent activation of microglia via NF-kappaB and AP-1 Co-regulation of COX-2 expression by S100B, IL-1beta and TNF-alpha. Neurobiology of aging, 2010. 31(4): p. 665-77.

28. Mukherjee, T.K., P.R. Reynolds, and J.R. Hoidal, Differential effect of estrogen receptor alpha and beta agonists on the receptor for advanced glycation end product expression in human microvascular endothelial cells. Biochimica et biophysica acta, 2005. 1745(3): p. 300-9.

29. de Arriba, S.G., et al., Advanced glycation endproducts induce changes in glucose consumption, lactate production, and ATP levels in SH-SY5Y neuroblastoma cells by a redoxsensitive mechanism. J Cereb Blood Flow Metab, 2003. 23(11): p. 1307-13.

30. Park, I.H., et al., Expression of a novel secreted splice variant of the receptor for advanced glycation end products (RAGE) in human brain astrocytes and peripheral blood mononuclear cells. Mol Immunol, 2004. 40(16): p. 1203-11.

31. Tanaka, N., et al., The receptor for advanced glycation end products is induced by the glycation products themselves and tumor necrosis factor-alpha through nuclear factor-kappa $B$, and by 17beta-estradiol through Sp-1 in human vascular endothelial cells. J Biol Chem, 2000. 275(33): p. 25781-90.

$4 \quad$ All work in this Biorvix publication remains the sole copyright of the named authors. 
32. Lee, F.T., et al., Interactions between Angiotensin II and NF-\{kappa\}B-Dependent Pathways in Modulating Macrophage Infiltration in Experimental Diabetic Nephropathy. J Am Soc Nephrol, 2004. 15(8): p. 2139-2151.

33. Coughlan, M.T., et al., Combination therapy with the advanced glycation end product crosslink breaker, alagebrium, and angiotensin converting enzyme inhibitors in diabetes: synergy or redundancy? Endocrinology, 2007. 148(2): p. 886-95.

34. Kislinger, T., et al., Receptor for advanced glycation end products mediates inflammation and enhanced expression of tissue factor in vasculature of diabetic apolipoprotein E-null mice. Arterioscler Thromb Vasc Biol, 2001. 21(6): p. 905-10.

35. Wendt, T.M., et al., RAGE drives the development of glomerulosclerosis and implicates podocyte activation in the pathogenesis of diabetic nephropathy. Am J Pathol, 2003. 162(4): p. 1123-37.

36. Oldfield, M.D., et al., Advanced glycation end products cause epithelial-myofibroblast transdifferentiation via the receptor for advanced glycation end products (RAGE). J Clin Invest, 2001. 108(12): p. 1853-63.

$5 \quad$ All work in this Biorvix publication remains the sole copyright of the named authors. 
A

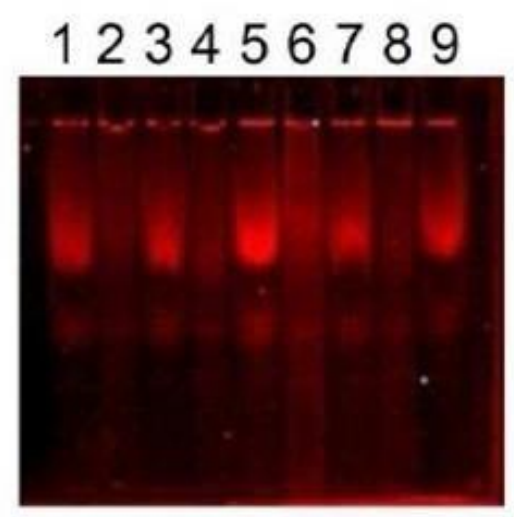

B

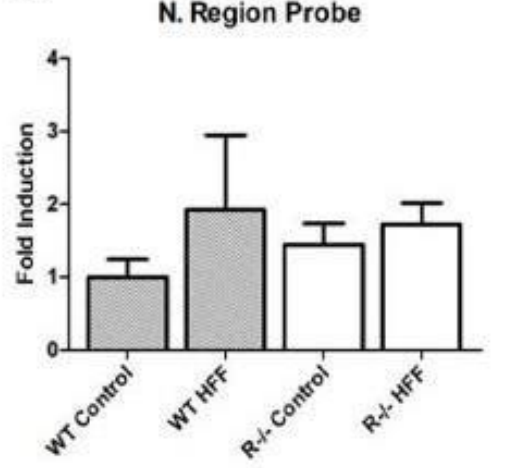

C ER- $\alpha$ ChIP

S. Region Probe

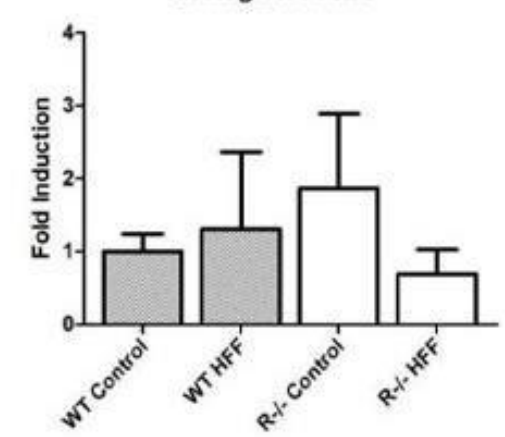

Supplementary Figure. Electromobility shift assay (EMSA) of kidney cortex DNA from Control mice. Lane 1 and 3. Construct 4. Lane 2 and 4. Construct 4. with RAGE Antibody Supershift, Lane 5 and 7. Construct 5. Lane 6 and 8. Construct 5. with RAGE Antibody Supershift. Lane 9. No DNA, RAGE Antibody Control. B-C Chromatin immunoprecipitation (ChIP) of DNA from kidney cortex of WT and RAGE deficient, Control and high fat fed mice. B: Interaction of ER- $\alpha$ bound chromatin in A. Region of the $5^{\prime}$ promoter region of RAGE. C: Interaction of ER- $\alpha$ bound chromatin in N. Region of the $5^{\prime}$ promoter region of the RAGE gene.

6 All work in this Biorvix publication remains the sole copyright of the named authors. 